\title{
Thank you for 2019
}

\section{Jon Karlsson ${ }^{1} \cdot$ Volker Musahl $^{2} \cdot$ Roland Becker $^{3} \cdot$ Michael T. Hirschmann $^{4}$}

Published online: 11 January 2020

(c) European Society of Sports Traumatology, Knee Surgery, Arthroscopy (ESSKA) 2020

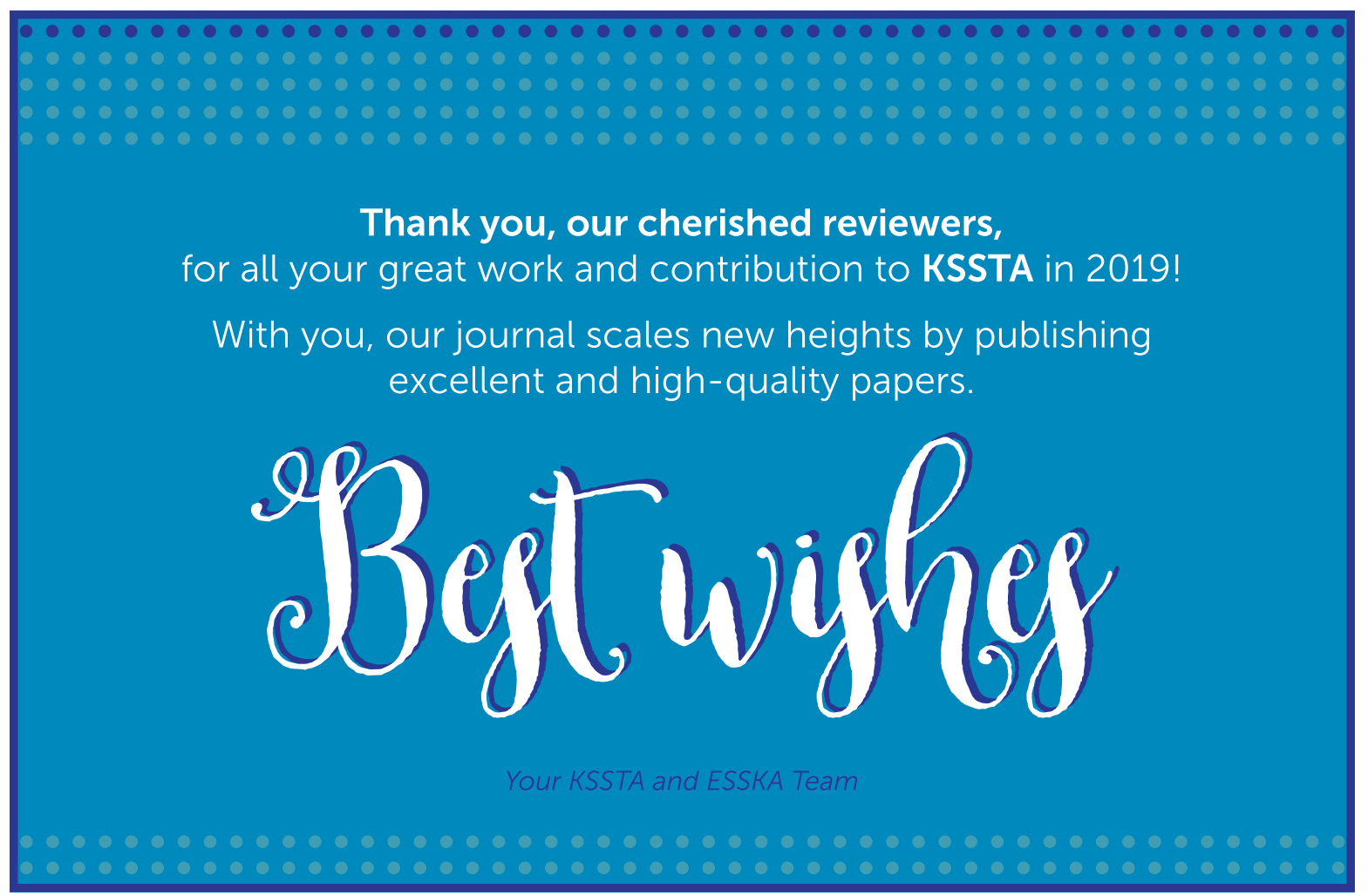

Jon Karlsson

jon.kssta@gmail.com

1 Department of Orthopaedics, Sahlgrenska Academy, University of Gothenburg, Sahlgrenska University Hospital, 43180 Mölndal, Gothenburg, Sweden

2 Department of Orthopaedic Surgery, Center for Sports Medicine, UPMC Rooney, Pittsburgh Medical Center, 3200 S Water Street, Pittsburgh, PA 15203, USA

3 Department of Orthopedics and Traumatology, Centre of Joint Replacement, Hospital Brandenburg, Medical School "Theodor Fontane", Hochstrasse 2, 14770 Brandenburg, Germany

4 Department of Orthopaedic Surgery and Traumatology, Kantonsspital Baselland (Bruderholz Liestal, Laufen), 4101 Bruderholz, Switzerland
Once again we are at the end of yet another year and it's time to say our customary thank you to all the dedicated persons who work for our journal. Firstly, thank you to all our reviewers who continuously help us with the peer-review process. Without our reviewers - and we have mentioned this many times - there would simply be no journal. Many or even most of you reviewers do such great work, by sending in high quality and timely reviews; both are very important. So, once more, thank you reviewers.

Our journal is soon turning 30, a grown-up, not a teenager any more. We have made some changes during this year. One of the most important changes is that we have two very qualified people who take care of business on a day-by-day basis at the Editorial Office in Luxembourg, 
Runeeta Rai and Simona Luparia. Both are highly qualified and experienced and of course, we would like to thank them for the work during the year. The Editorial Office is our face towards the outside world, therefore, it is important that we maintain high quality, professionalism and care about the authors who submit their manuscripts.

We would also like to use this opportunity to thank our publisher, Springer, for an extremely good collaboration during a long period of time. Especially, we want to thank Gabriele Schröder who has been such a great support to the journal through the years. Now, David Stanmore will work closely with us and we welcome this new collaboration.

During 2019, ESSKA expanded the scope of our sister journal, JEO (Journal of Experimental Orthopedics), to include clinical studies. JEO will now work even more closely with KSSTA to cover similar topics. One of our longlasting Associate Editors of KSSTA, Stefano Zaffagnini, is in the lead for JEO as its editor-in-chief and we would like to thank him for his great contribution to KSSTA and wish him luck in his new position. We look forward to even more fruitful collaboration in the future.

There have been a few changes at the Associate Editor level; as both Stefano and Rainer Siebold have moved on. Thanks to both of you. New persons, all very qualified, have taken your place; Eduard Alentorn-Geli (Spain), Sverre Löken (Norway) and Tim Spalding (UK) are our new Associate Editors and you are all welcome on the team.

We also thank all the guest editors who worked hard to bring out great Special Theme Issues in 2019!

We have toiled hard to improve our social media presence on Facebook and our new web-page, etc. We would like to thank Elmar Herbst and Mahmut Enes Kayaalp for their untiring work, especially editing video-clips that improve the pedagogical content of web-publications.

Of course, we would also like to thank the ESSKA Board and the ESSKA Executive Office for the continuous good collaboration during 2019. We also look forward to 2020 there are challenges, but in many ways business will be as usual, KSSTA will continue as a printed journal, with more than 300 pages each month.

\author{
Aagaard, Henrik \\ Abrams, Jeffrey \\ Achtnich, Andrea \\ Adachi, Nobuo \\ Agricola, Rintje \\ Ahldén, Mattias \\ Åhlén, Martina \\ Ahmad, Sufian \\ Ahrens, Philipp \\ Alaia, Michael \\ Ambrozic, Bogdan \\ Andrish, Jack \\ Antinolfi, Pierluigi \\ Araki, Daisuke \\ Araujo, Paulo \\ Arendt, Elizabeth \\ Arrigoni, Paolo \\ Attal, Rene \\ Augustsson, Jesper \\ Bae, Ji-Hoon \\ Balcarek, Peter \\ Balke, Maurice \\ Baranto, Adad \\ Barenius, Björn \\ Barfod, Kristoffer \\ Barth, Johannes \\ Batailler, Cecile \\ Bauer, Thomas \\ Baums, Mike \\ Beaufils, Philippe
}

Becher, Christoph

Beckmann, Johannes

Behrend, Henrik

Beitzel, Knut

Berruto, Massimo

Beyzadeoglu, Tahsin

Bilge, Onur

Bilgic, Erkal

Bitsch, Rudi

Bjørnsson Hallgren, Hanna

Blønd, Lars

Boettner, Friedrich

Bonanzinga, Tommaso

Bosch, Ulrich

Brandl, Georg

Braun, Sepp

Briem, Kristín

Brittberg, Mats

Brockmeyer, Matthias

Brorsson, Annelie

Brouwer, Reinoud W.

Budhiparama, Nicolaas

Bulgheroni, Paolo

Burnham, Bruce

Burnham, Jeremy

Calder, James

Carmont, Mike

Castellet, Enric

Cavaignac, Etienne

Cerciello, Simone

\author{
Chang, Chong Bum \\ Chen, Antonia \\ Cheng, Cheng-Kung \\ Cift, Hakan \\ Clatworthy, Mark \\ Clauss, Martin \\ Compagnoni, Riccardo \\ Condello, Vincenzo \\ Conteduca, Fabio \\ Coughlin, Ryan \\ Cucchi, Davide \\ Dahmen, Jari \\ Dalmau-Pastor, Miki \\ De Bo, Thomas \\ de Casas, Ricardo \\ De Girolamo, Laura \\ De Gori, Marco \\ de SA, Darren \\ Deep, Kamal \\ Defoort, Koen \\ Deie, Masataka \\ Dejour, David \\ Demey, Guillaume \\ Denti, Matteo \\ Devitt, Brian \\ Diamond, Laura \\ Diermeier, Theresa \\ Dietrich, Tobias \\ Domnick, Christoph \\ Donell, Simon
}


Doral, Mahmut

Draenert, Klaus

Drews, Björn

Drobnic, Matej

Drogset, Jon

Dyrna, Felix

Einarsson, Fredrik

Ekås, Guri

Endres, Nathan

Erdil, Mehmet

Eriksson, Bengt

Eriksson, Karl

Ernlund, Lucio

Eslam Pour, Aidin

Espregueira-Mendes, João

Ettinger, Max

Euler, Simon A.

Eygendaal, Denise

Familiari, Filippo

Farr, Jack

Fauno, Peter

Fehske, Kai

Ferretti, Mario

Feucht, Matthias

Filardo, Giuseppe

Fink, Christian

Fleaca, Radu

Friel, Nicole

Gelber, Pablo

Gerhardt, Christian

Gersoff, Wayne

Gill, Thomas

Giron, Francesco

Glasbrenner, Johannes

Glazebrook, Mark

Gliatis, John

Gobbi, Alberto

Gokeler, Alli

Gomez Barrena, Enrique

Gomoll, Andreas

Grassi, Alberto

Grävare Silbernagel, Karin

Greenberg, Elliot

Grindem, Hege

Grote, Stefan

Gunther, Daniel

Haasper, Carl

Hage, William

Hägglund, Martin

Halder, Andreas

Hamrin Senorski, Eric

Hams, Andrea

Harilainen, Arsi
Harrer, Joerg

Heesterbeek, Petra

Hess, Silvan

Heuberer, Philipp

Hiemstra, Laurie

Hinarejos, Pedro

Hirschmann, Anna

Hochrein, Alfred

Hofmann, Siegfried

Hogan, MaCalus

Hoshino, Yuichi

Howell, Stephen

Hulet, Christophe

Hutchinson, Mark

Imhoff, Andreas

Inderhaug, Eivind

Iosifidis, Michael

Iriuchishima, Takanori

Iseki, Tomoya

Ivarsson, Andreas

Izadpanah, Kaywan

Janssen, Rob

Jarvela, Timo

Jayachandran, Jayadeep

Jelic, Mislav

Jenny, Jean-Yves

Jeong, Jae-Heon

Jerosch, Joerg

Jiang, Kevin

Kalawadia, Jay

Karahan, Mustafa

Kartus, Jüri

Kay, Jefferey

Kennedy, John

Kessler, Oliver

Khan, Moin

Khoschnau, Shwan

Kittl, Christoph

Knutsen, Gunnar

Kobayashi, Koichi

Kocaoglu, Baris

Koga, Hideyuki

Koh, Jason

Kostogiannis, Ioannis

Krogsgaard, Michael

Kroll, Artur

Krutsch, Werner

Kundra, Rik

LaPrade, Robert

Larrainzar-Garijo, Ricardo

Laver, Lior

Lawhorn, Keith

Lee, Dae-Hee
Leeuw, Peter

Lefevre, Nicolas

Lertwanich, Pisit

Levy, Ofer

Lichtenberg, Sven

Liebensteiner, Michael

Lin, Albert

Lind, Martin

Lindahl, Anders

Lodhia, Parth

Lopomo, Nicola

Lorbach, Olaf

Lund, Bent

Lundberg, Mari

Lützner, Jörg

Lyman, Stephen

Macalena, Jeffrey

Macdonald, Peter

Maffulli, Nicola

Magnitskaya, Nina

Mahirogullari, Mahir

Makridis, Konstantinos

Marcheggiani Muccioli, Giulio Maria

Margheritini, Fabrizio

Martetschläger, Frank

Martinek, Vladimir

Martinez, Inigo

Massin, Philippe

Mathis, Dominic

Matsumoto, Tomoyuki

Mauro, Craig

Mayr, Hermann Otto

Meknas, Khaled

Memon, Muzammil

Moatshe, Gilbert

Mogos, Stefan

Monllau, Juan

Moser, Lukas

Moström, Eva

Mouton, Caroline

Mueller, Andreas

Muellner, Thomas

Müller, Sebastian

Muneta, Takeshi

Næsheim, Truls Straume

Nagai, Kanto

Nakamae, Atsuo

Nakamura, Norimasa

Nebelung, Wolfgang

Nelitz, Manfred

Niemeyer, Philipp

Nilsson-Helander, Katarina

Nyland, John 


Ollivier, Matthieu
Orth, Patrick
Owesen, Christian
Pagenstert, Geert
Pap, Géza
Patel, Rahul
Patralekh, Mohit
Patzer, Thilo
Pauyo, Thierry
Pearce, Christopher
Pederzini, Luigi
Peng, Yun
Pérez Prieto, Daniel
Persson, Andreas
Peterson, Devin
Petri, Maximilian
Petrigliano, Frank
Picard, Frederic
Pietsch, Martin
Piscaer, Tom
Plachel, Fabian
Plancher, Kevin
Pogorzelski, Jonas
Prodromos, Chadwick
Pujol, Nicolas
Randelli, Pietro
Randelli, Filippo
Randsborg, Per-Henrik
Rath, Bjoern
Riviere, Charles
Roberti di Sarsina, Tommaso
Rodeo, Scott
Roe, Justin
Roessler, Philip
Rosso, Claudio
Rothrauff, Benjamin
Røtterud, Jan Harald

\begin{tabular}{|c|c|}
\hline Ruiz Iban, Miguel & Takayama, Koji \\
\hline Russu, Octav & Thompson, Simon \\
\hline Sadoghi, Patrick & Thomsen, Peter \\
\hline Saffarini, Mo & Thorey, Fritz \\
\hline Salomonsson, Björn & Tischer, Thomas \\
\hline Samitier, Gonzalo & Tompkins, Marc \\
\hline Sansone, Mikael & Torres-Claramunt, Raul \\
\hline Saris, Daniel & Tranberg, Roy \\
\hline Schiavone Panni, Alfredo & Tscholl, Philippe \\
\hline Schliemann, Benedikt & Tsepis, Elias \\
\hline Schröter, Steffen & Van Arkel, Ewould \\
\hline Schwab, Pierre-Emmmanuel & van Bergen, Christiaan \\
\hline Seijas, Roberto & van den Bekerom, Michel P. J. \\
\hline Seil, Romain & van der Meijden, Olivier \\
\hline Shearman, Alex & van Eck, Carola \\
\hline Sheean, Andrew & Van Giffen, Nicolien \\
\hline Sherman, Seth & Vasso, Michele \\
\hline Shimozono, Yoshiharu & Ventura, Alberto \\
\hline Siebenlist, Sebastian & Violante, Bruno \\
\hline Siebold, Rainer & Vioreanu, Mihai \\
\hline Simion, Luminita & Waibl, Bernhard \\
\hline Sivertsen, Einar Andreas & Walsh, William \\
\hline Skråmm, Inge & Weber, Patrick \\
\hline Smolen, Daniel & Webster, Kate \\
\hline Soler, Francesc & Werthel, Jean-David \\
\hline Sørensen, Anne Kathrine & Westin, Maria \\
\hline Spahn, Gunter & Willwacher, Steffen \\
\hline Spennacchio, Pietro & Wilmes, Philippe \\
\hline Stalman, Anders & Wolf, Brian \\
\hline Stärke, Christian & Wouters, Diederick \\
\hline Steensen, Robert & Younger, Alastair \\
\hline Stoehr, Amelie & Zaffagnini, Stefano \\
\hline Stufkens, Sjoerd & Zampeli, Frantzeska \\
\hline Sullivan, Justin & Zeisig, Eva \\
\hline Sundemo, David & Zellers, Jennifer \\
\hline Svantesson, Eleonor & Zupanc, Oskar \\
\hline
\end{tabular}

Publisher's Note Springer Nature remains neutral with regard to jurisdictional claims in published maps and institutional affiliations. 